\title{
APOPTOSIS AND BCL-2 EXPRESSION IN IRRADIATED LUNGS AND THE EFFECT OF PENTOXIFYLLINE
}

\author{
Jan Österreicher ${ }^{1}$, Michal Králik ${ }^{1}$, Leoš Navrátil ${ }^{2}$, Jiřina Vávrová ${ }^{1}$, Jiř̌ Škopek ${ }^{2}$, Jiř̌ Knížek ${ }^{1}$, Aleš Macela ${ }^{1}$ \\ Purkyně Military Medical Academy, Hradec Králové: Department of Radiobiology and Immunology ${ }^{1}$; Charles University \\ in Prague, $1^{\text {st }}$ Medical Faculty: Department of Biophysics ${ }^{2}$
}

\begin{abstract}
Summary: We measured number of bcl-2, apoptotic, neutrophil, and surfactant apoprotein D (SP-D) positive cells in irradiated rat lungs during different time points after the sublethal whole-thorax irradiation of rats. We also investigated the influence of pentoxifylline (PTX) therapy on these markers. Wistar rats were given 15 Gy thoracic irradiation and PTX $(35 \mathrm{mg} / \mathrm{kg}$ ) twice a week. Animals were examined histologically and imunohistochemically at intervals from 1-12 weeks. In non-treated rats compared with treated rats, bcl-2 expression was significantly inhibited from 4 weeks after irradiation. A higher apoptosis presence in non-treated rats from 4 weeks was found and apoptosis development in PTX-treated animals was delayed and started 8 weeks after irradiation. Similar differences were measured during neutrophil granulocytes examination. Neutrophil penetration in non-treated rats was found 5 weeks after irradiation in contrast to the RP onset of PTX-treated animals 8 weeks after irradiation. The number of SP-D positive cells in non-treated rats observed until 5 weeks after irradiation was higher than in the control group. PTX-treated animals expressed higher number of SP-D positive cells during the whole experiment than the control group. We suggest that apoptosis is linked to neutrophil granulocyte actions during the RP onset and that PTX-therapy causes diminished inflammation development.
\end{abstract}

Key words: Irradiation; Lung; Pentoxifylline; Apoptosis; Bcl-2

Abbreviations: RP - radiation pneumonitis; ARDS - adult respiratory distress syndrome; DAD - diffuse alveolar damage; TNF - tumor necrosis factor; IL - interleukin; S.E.M - standard error of mean. PTX - pentoxifylline; PBS - phosphatebuffered saline; cAMP - cyclic adenosine monophosphate; ATP - adenosine triphosphate; DXM - dexamethasone; IFN-interferone; SP-D- surfactant apoprotein D.

\section{Introduction}

Radiation pneumonitis (RP) is defined as an exudative inflammation which occurs as a result of ionizing radiation (5) and it is considered as an alveolitis resulting from damage to pneumocytes and the endothelial cells (13). This condition is termed adult respiratory distress syndrome (ARDS), sometimes as diffuse alveolar damage (DAD; 16). $\mathrm{RP}$ is usually manifested from 2 to 6 months after irradiation of the lungs with a single dose of $7 \mathrm{~Gy}$ or more (2). It involves changes in the irradiated areas only (29).

Apoptosis (programmed cell death) is important for cellular homeostasis in a variety of tissues. The mechanisms regulating apoptosis are complex and involve the interaction of nuclear and cytoplasmic proteins. Recently, the sensitivity of tissues to apoptosis induced by a variety of external stimuli has been closely linked to the intracellular concentrations of a family of cell-death regulators, which include bcl-2 (14). High levels of bcl-2 protect against apoptosis through inhibition of caspases activation, the key enzymes of apoptosis (7). Guinee et al. (15) and Bardales et al. (1) have shown that apoptosis is demonstrable in type II pneumocytes in DAD and not in normal lung tissues. In this regard, the action of bcl-2 in DAD is potentially analogous to its action in lymphomas and carcinomas: the prevention of apoptosis (19). Fuks et al. (8) first described apoptosis in irradiated lung tissue by the DNA terminal transferase nick-end translation method. This method revealed time and dose-dependent apoptosis mainly in endothelial and mesothelial cells at 1 week after irradiation.

Pentoxifylline (PTX) ameliorates radiation-induced histological changes in irradiated lungs (25). Funk et al. (9) observed the in vitro influence of cytokine production after the addition of PTX alone and of PTX in combination with DXM during experiments with human peripheral mononuclear cells, which were stimulated by phytohemaglutinin. These results suggest that PTX inhibits the production of TNF- $\alpha$, IL-2, and IFN- $\gamma$, proinflammatory cytokines. In recent clinical studies, Silva et al. (27), Koo et al. (18) in rat models and Noel et al. (21) in clinical studies independent- 
ly confirmed inhibition of TNF- $\alpha$ and other pro-inflammatory cytokines during PTX-therapy. If the hypothesis of RP development via the overexpressed pro-inflammatory cytokine cascade (TNF- $\alpha$, IL-1 and others) is correct (26), then the therapeutic period influencing the expression of cytokines starts immediately after irradiation because an increased production of proinflammatory cytokines was found immediately after irradiation.

In this study, we sought to test the effect of PTX administered from day 1 to day 84 ( 12 weeks) after irradiation and to study the link with bcl-2 expression. We investigated apoptosis by the caspase cleavage product of cytokeratin 18 as well as the number of neutrophils and surfactant apoprotein $\mathrm{D}$ positive cells in the irradiated lungs by computer image analysis.

\section{Material and Methods}

Male Wistar rats (Konarovice, Czech Republic) aged 812 weeks and weighing 150-200 g were given local thoracic irradiation using a ${ }^{60} \mathrm{Co}$ unit (Chisotron Chirana) at a dose rate of $1.0 \mathrm{~Gy} / \mathrm{min}$, target distance $1 \mathrm{~m}$. The animals were slightly anaesthetised before irradiation using a mixture of one volume of Rometar (Spofa Company, Prague), 3 volumes of Narkamon (Léčiva Company, Prague) and 12 volumes of physiological saline. This solution was injected intramuscularly at $10 \mathrm{ml} / \mathrm{kg}$. Local thoracic irradiation was performed in a jig in which a $10 \mathrm{~cm}$ thick layer of lead reduced the dose to other parts of the body to around $2-3 \%$ of the lung dose (30).

Three groups of rats were used: Group S was given physiological saline only. Group PTX 35 was given pentoxifylline (Pentilin, Krka, $100 \mathrm{mg} / 5 \mathrm{ml}$ ampoules) diluted with physiological saline and administered at a dose of 35 $\mathrm{mg} / \mathrm{kg}$; while Group C involved sham-irradiated controls.

PTX and saline were injected subcutaneously twice a week.

Total number of examined rats was 90. Group C contained 6 non-irradiated rats. An each irradiated group (Group S and PTX 35) included 7 irradiated subgroups by 6 animals in each subgroup. Total number of 84 rats (except group C) was irradiated with 15 Gy and treated $1,2,3,4,5,8$ and 12 weeks after irradiation. This radiation dose resulted in significant pneumonitis and fibrosis but no mortality up to 30 weeks, whereas 16 Gy resulted in over $50 \%$ mortality 6 weeks after irradiation (30). The first drug administration was given $2 \mathrm{~h}$ after irradiation and the last at two days before the animals were sacrificed.

\section{Histologic Examination}

Rats were killed by cervical dislocation and lung histology examined 1,2,3,4,5,8 and 12 weeks after irradiation; controls were killed at 12 weeks. Measured marker levels in 6 control rats 12 weeks after irradiation were used as reference values, except in the M30 and bcl-2 examinations. During dissection, the lungs were carefully fixed per trachea with $10 \%$ neutral buffered formalin, embedded into paraffin and $4 \mathrm{~mm}$ thick tissue sections were cut, stained with chloracetate esterase to detect neutrophil granulocytes and Gramm's staining for bacterial infection.

Immunohistochemical examinations for bcl-2, direct detection of apoptotic cells, and surfactant apoprotein D positive cells were performed with a standard immunoperoxidase technique. After blocking endogenous peroxidase activity for $20 \mathrm{~min}$, tissue sections were incubated with individual polyclonal antibodies (anti-bcl-2 rabbit antibody, Santa Cruz, CA, USA) diluted 1:300 in phosphate-buffered saline (PBS, pH 7.2) for $24 \mathrm{~h}$ at $4^{\circ} \mathrm{C}$, and then washed three times in PBS. The slides for bcl-2 detection were then incubated with a horseradish peroxidase-coupled anti-rabbit antibody (Santa Cruz, CA, USA) for 45 minutes at $37^{\circ} \mathrm{C}$. Excess antibodies were washed off with PBS. Finally, a $0.05 \%$ 3.3-diaminobenzidine tetrahydrochloride chromogen solution (Sigma, Prague, Czech Republic) in PBS containing $0.02 \%$ hydrogen peroxide was added for $10 \mathrm{~min}$ to visualise the antigen-antibody complex in situ.

A M30 Cytodeath kit (Roche Diagnostics, Mannheim, Germany) was employed for the detection of apoptotic cells. The staining procedure was as for bcl-2, whereas incubation with the primary antibody was for 1 hour at room temperature and incubation with the anti-mouse secondary horseradish peroxidase-coupled antibody for 30 minutes at room temperature.

Surfactant apoprotein D was detected as with M30 Cytodeath, whereas samples were put in citrate saline $(\mathrm{pH} 6.0)$ and twice heated in a microwave $(750 \mathrm{~W})$ for a period of 5 minutes. Samples were then incubated with the mouse monoclonal anti-surfactant apoprotein D antibody (clone VI F11, from Prof. Michael Kasper, Technische Universität, Dresden, Germany) diluted 1:10 in PBS for $1 \mathrm{~h}$ at room temperature.

As negative controls were used samples stained without primary antibodies.

\section{Measurement of bcl-2, $M$ 30, and SP-D positive cell numbers}

Immunohistochemical samples were evaluated using an IMT-2 light microscope (Olympus Company, Prague) and computer image analysis (Image Pro, Media Cybernetics, MD, USA). Ten randomly selected viewing fields with a size of $10744,32 \mu \mathrm{m}^{2}$ without bronchi and large vessels from each sample were evaluated at a 600 fold original magnification.

\section{Data Processing}

The Mann-Whitney test was used for statistical analysis, giving a mean $\pm 2 \times$ SEM.

\section{Results}

The following bcl-2 positive cells were found: type-II pneumocytes, fibroblasts, fibrocytes, endothelial cells and 
Tab. 1: Average number of bcl-2 positive cells $\pm 2 \mathrm{x}$ SEM in the lungs.

\begin{tabular}{|l|c|c|c|c|c|c|c|}
\hline & 1 week & 2 weeks & 3 weeks & 4 weeks & 5 weeks & 8 weeks & 12 weeks \\
\hline Group S & $13.57 \pm 2.12^{3}$ & $18.65 \pm 2.36^{2}$ & $14.75 \pm 3.00^{3}$ & $10.78 \pm 2.64^{3}$ & $15.30 \pm 2.72^{3}$ & $10.80 \pm 1.52^{3}$ & $17.42 \pm 2.88^{2}$ \\
\hline Group PTX 35 & $11.92 \pm 2.20^{3}$ & $19.70 \pm 3.32^{2}$ & $19.53 \pm 4.32^{2}$ & $29.25 \pm 5.20^{\mathrm{c}}$ & $34.08 \pm 3.52^{\mathrm{c}}$ & $29.55 \pm 4.01^{\mathrm{c}}$ & $31.80 \pm 4.24^{\mathrm{c}}$ \\
\hline Group C & & & & & & & $27.78 \pm 4.52$ \\
\hline
\end{tabular}

Probability of value differences to Group C 12 weeks after irradiation: $\mathrm{p}<0.05-{ }^{1} ; \mathrm{p}<0.01-{ }^{2} ; \mathrm{p}<0.001-{ }^{3}$.

Probability of value differences between Group S and Group PTX 35 in the same time interval: $p<0.05-{ }^{a} ; p<0.01-{ }^{b}$; $\mathrm{p}<0.001-\mathrm{c}$.

Tab. 2: Average number of apoptotic cells \pm 2 x SEM in the lungs.

\begin{tabular}{|l|c|c|c|c|c|c|c|}
\hline & 1 week & 2 weeks & 3 weeks & 4 weeks & 5 weeks & 8 weeks & 12 weeks \\
\hline Group S & $0.18 \pm 0.12$ & $1.63 \pm 0.88$ & $0.12 \pm 0.16^{1}$ & $4.25 \pm 2.04^{3}$ & $17.05 \pm 2.68^{3}$ & $10.25 \pm 3.08^{3}$ & $8.43 \pm 1.92^{3}$ \\
\hline Group PTX 35 & $3.38 \pm 1.08^{\mathrm{c}}$ & $1.67 \pm 1.03$ & $0.2 \pm 0.16$ & $0.08 \pm 0.12^{\mathrm{c}}$ & $0.02 \pm 0.04^{\mathrm{c}}$ & $2.67 \pm 1.12^{\mathrm{a}}$ & $3.83 \pm 1.28^{3 \mathrm{c}}$ \\
\hline Group C & & & & & & & $0.35 \pm 0.28$ \\
\hline
\end{tabular}

Probability of value differences to Group C 12 weeks after irradiation: $p<0.05-{ }^{1} ; \mathrm{p}<0.01-{ }^{2} ; \mathrm{p}<0.001-{ }^{3}$.

Probability of value differences between Group S and Group PTX 35 in the same time interval: $p<0.05-{ }^{a} ; p<0.01-{ }^{b}$; $\mathrm{p}<0.001-\mathrm{c}$.

Tab. 3: Average number of neutrophils in the lungs \pm 2 x S.E.M.

\begin{tabular}{|l|c|c|c|c|c|c|c|}
\hline & 1 week & 2 weeks & 3 weeks & 4 weeks & 5 weeks & 8 weeks & 12 weeks \\
\hline Group S & $0.09 \pm 0.05$ & $0.07 \pm 0.04$ & $0.11 \pm 0.07$ & $0.07 \pm 0.07$ & $1.24 \pm 0.34^{1}$ & $4.80 \pm 0.60^{3}$ & $5.95 \pm 0.56^{3}$ \\
\hline Group PTX 35 & $0.11 \pm 0.06$ & $0.21 \pm 0.07$ & $0.1 \pm 0.06$ & $0.08 \pm 0.04$ & $0.16 \pm 0.06^{\mathrm{a}}$ & $1.96 \pm 0.27^{3 \mathrm{~b}}$ & $3.41 \pm 0.45^{3 \mathrm{c}}$ \\
\hline Group C & & & & & & & $0.46 \pm 0.11$ \\
\hline
\end{tabular}

Probability of value differences to Group C: $\mathrm{p}<0.05-{ }^{1} ; \mathrm{p}<0.01-{ }^{2} ; \mathrm{p}<0.001-{ }^{3}$.

Probability of value differences between Group S and Group PTX 35 in the same time interval: $p<0.05-{ }^{a} ; p<0.01-{ }^{b}$; $\mathrm{p}<0.001-\mathrm{c}$.

Tab. 4: Average number of SP-D positive cells in the lungs \pm 2 x S.E.M.

\begin{tabular}{|l|c|c|c|c|c|c|c|}
\hline & 1 week & 2 weeks & 3 weeks & 4 weeks & 5 weeks & 8 weeks & 12 weeks \\
\hline Group S & $9.02 \pm 1.00^{3}$ & $10.48 \pm 0.90^{3}$ & $10.05 \pm 0.99^{3}$ & $8.48 \pm 1.32^{\mathbf{1}}$ & $7.85 \pm 0.76^{\mathbf{2}}$ & $7.23 \pm 0.87$ & $6.74 \pm 0.96$ \\
\hline Group PTX 35 & $12.40 \pm 0.92^{3 \mathbf{c}}$ & $9.55 \pm 0.87^{\mathbf{3}}$ & $14.35 \pm 1.40^{\mathbf{3 c}}$ & $12.33 \pm 1.69^{3 \mathbf{b}}$ & $8.75 \pm 0.96^{3}$ & $9.53 \pm 0.83^{3 \mathbf{c}}$ & $9.10 \pm 1.20^{\mathbf{2 b}}$ \\
\hline Group C & & & & & & & $6.38 \pm 0.89$ \\
\hline
\end{tabular}

Probability of value differences to Group C: $\mathrm{p}<0.05-{ }^{1} ; \mathrm{p}<0.01-{ }^{2} ; \mathrm{p}<0.001-{ }^{3}$.

Probability of value differences between Group S and Group PTX 35 in the same time interval: $p<0.05-{ }^{a} ; \mathrm{p}<0.01-{ }^{b}$; $\mathrm{p}<0.001-\mathrm{c}$.

macrophages. Epithelial cells of the bronchial tree were also positive but the bronchi were not evaluated. Apoptosis was detected in the epithelium, in type-II pneumocytes and occasionally macrophages (after phagocytosis) and endothelial cells. SP-D positive staining in type-II pneumocytes, macrophages, and unexamined bronchial epithelial cells was observed. No infectious agents were found.

In Group S, 3 animals died, 2 at 4 weeks and 1 at 12 weeks after irradiation. One rat from the PTX-administered group died at 12 weeks after irradiation.

\section{The bcl-2 expression measurement}

Significantly higher values of Group C compared with Group S were measured 12 weeks after irradiation. The effect of PTX therapy was expressed by a higher number of positive staining cells beyond 4 weeks than in Group S.

\section{Apoptotic cell measurement}

Significantly higher values of this marker of Group C compared with Group S were measured 12 weeks after irradiation. In Group PTX 35, the effect of therapy expressed 


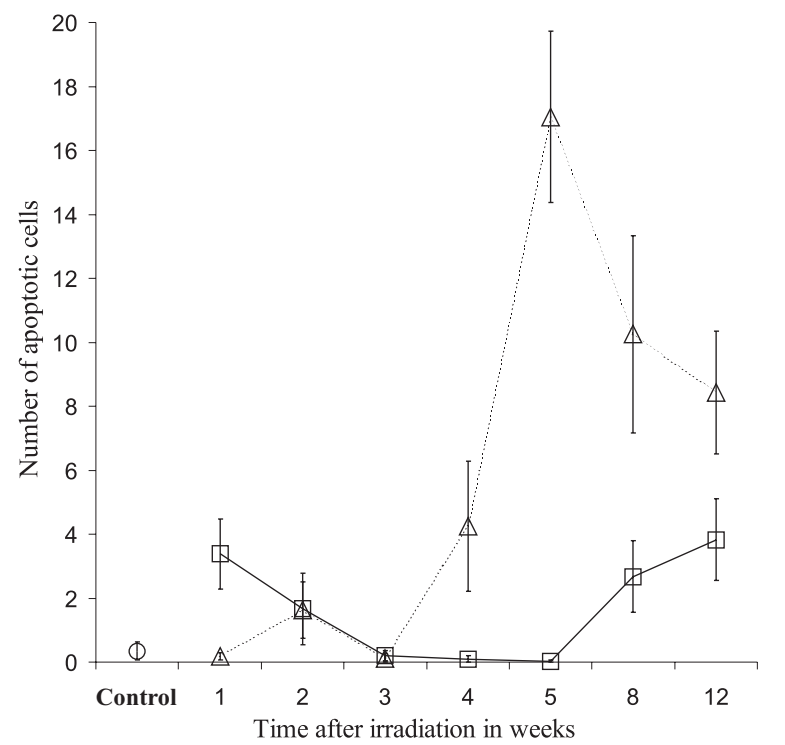

Fig. 1: Average number of apoptotic cells in the lungs after 15 Gy irradiation. The error bars are in the form of $95 \%$ confidence intervals. ( $\Delta$ - Group S, $\square$ - Group PTX 35, o Group C).

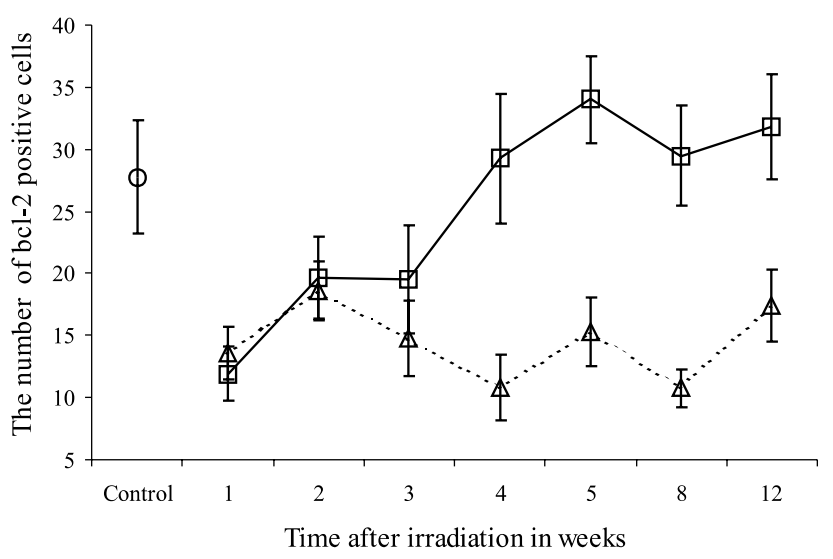

Fig. 2: Average number of bcl-2 positive cells in the field in the lungs after $15 \mathrm{~Gy}$ irradiation. The error bars are in the form of $95 \%$ confidence intervals. ( $\Delta$ - Group S, $\square$ - Group PTX 35, o - Group C).

by the number of apoptotic cells was found at 1 week and from 4 weeks after irradiation to the end of the experiment in comparison with Group S.

\section{The number of neutrophil granulocytes}

From 5 weeks after irradiation to the end of the experiment a significantly higher number of neutrophils was found in Group S than in Group C. The penetration of neutrophils in the lungs of PTX-treated rats was delayed, a higher number of neutrophils were observed 8 weeks after irradiation, significantly lower than in Group S.

\section{The number of SP-D positive cells}

Until 5 weeks after irradiation, a significantly higher number of SP-D positive cells were measured in Group $\mathrm{S}$ than in Group C. In the lungs of PTX-treated rats during the whole experiment, a higher number of SP-D immunoreactive cells were detected than in Group C. The effect of PTX therapy was observed in all time intervals, except 2 and 5 weeks after sublethal irradiation.

\section{Discussion}

Many of the known etiologies of diffuse alveolar damage, including radiation, may cause DNA damage and thereby induce apoptosis $(13,17)$.

In animal models, DAD is associated with the generation of free radicals, which include the superoxide anion $\left(\mathrm{O}_{2}^{-}\right)$, hydrogen peroxide $\left(\mathrm{H}_{2} \mathrm{O}_{2}\right)$, the hydroxyl radical $(\mathrm{OH} \bullet)$, and singlet oxygen $\left(\mathrm{O}_{2} \bullet\right)(6,23)$. These free radicals have been shown to induce apoptosis, presumably through damage to DNA (32). Korsmeyer et al. (20) postulated that free radical damage and its potential to induce apoptosis are regulated by the intracellular levels particularly of bcl-2. In this regard, bcl-2 has been localized to mitochondria, nuclear membranes, and the endoplasmic reticulum, which are sites where free radicals are generated (20). In DAD, the absence of bcl-2 expression in type IIpneumocytes suggest that these cells are vulnerable to the effects of free radical-induced apoptosis (14). Guinee et al. (15) have shown that apoptosis in DAD is associated with the expression of p53 and WAF1, suggesting that DNA damage and p53-dependent apoptosis may contribute to the pathogenesis and/or evolution of this disorder.

In our study, at 1 week after irradiation of the physiological saline-administered animals, an inhibition of bcl-2 followed by the presence of the apoptotic cells in the next time interval were seen. We assume that the presence of the apoptotic cells early after irradiation has a relationship to the action of the radiation-induced free radicals. Apoptotic cells increased significantly beyond 4 weeks after irradiation, one week before penetration of the neutrophils. On the other hand, the relationship between inflammation and apoptosis phenomena might be bilateral. Apoptotic cells and bodies are primarily removed by non-professional phagocytes, e.g. type II pneumocytes in the lungs. However, if they are not readily removed by phagocytosis, they undergo "secondary necrosis". During the secondary necrosis, the lysosomes rupture and release hydrolytic enzymes into the cytoplasm causing further destruction of the internal components including the plasma membrane. Once the plasma membrane loses integrity, the cell lyzes, releasing hydrolytic enzymes into the extracellular space and guaranteeing an inflammatory response with possible tissue damage (12).

At present, PTX is considered to be a preparation with various immunomodulatory effects which were found in vitro in plasmatic concentrations as low as $10 \mu \mathrm{g} / \mathrm{ml}$ (9) 
and $20 \mathrm{mg} / \mathrm{kg} /$ day per os in the rat model (27). The main effect of PTX in immune reactions is a decrease in the production of TNF- $\alpha(9,22)$ at the protein and as well as the mRNA level (3). Because of this effect, we used pentoxifylline for the treatment of post-irradiation apoptotic changes. Ward et al. (31) reported that PTX had only a small beneficial effect on a radiation-induced lung injury in a rat by some indices such as lung wet weight and protein content, but not by hydroxyproline content, the activity of the lung angiotensin converting enzyme and the plasminogen activator. From their results it follows that the PTX effect is not only through the pulmonary endothelial function, but also via other mechanisms.

The beneficial effects of repeated applications of PTX, DXM and their combination to sublethally irradiated mice were noted (25). PTX diminishes and/or delays the neutrophil granulocyte penetrations through the vascular wall in the lungs and the interstitial edema intensity during the RP onset $(24,25)$. In addition, it has been found in the Paraquat-affected tissue culture model formed by isolated pulmonary cells (28) that PTX reduces the production of oxygen radicals and scavenges free radicals.

The observed apoptosis inhibition during the RP onset phase in PTX-treated rats may have been caused via PTXinduced TNF- $\alpha$ inhibition and subsequently a lower inflammatory response in irradiated lungs. Moreover, TNF- $\alpha$ is one of the apoptotic inductors (4).

PTX-mediated overproduction of cAMP (3) and subsequently ATP (10) after phosphodiesterase inhibition may play a synergic role to stimulate surfactant secretion (11). Our results of surfactant apoprotein D positive cells show that PTX facilitates surfactant production in our model.

We suggested that apoptosis of type II-pneumocytes in our model is linked to inflammation onset after thoracic irradiation. Moreover, PTX therapy would cause a delayed and diminished radiation-induced inflammatory response in the lungs.

Further investigation of the early molecular postradiation pulmonary changes with cell identifications will be the objective of our work.

\section{Conclusion}

The reduced and delayed expression of apoptosis in irradiated lungs caused by PTX might be used as an effective tool for inhibition of radiation-induced changes of lungs. The inflammatory process and apoptosis in irradiated lungs are closely-related phenomena with a possible bilateral influence.

\section{Acknowledgements}

We would like to thank Prof. Dr. Michael Kasper for his rat reactive anti-SP-D antibody and we thank Mrs. Šárka Průchová and Hana Buzková for their skillful assistance. This study has been supported by MO 66020398127, MO 02031100002, and FJ MSM 111100005 grants.

\section{References}

1. Bardales RH, Xie S, Schaefer RF et al. Apoptosis is a major pathway responsible for the resolution of type II pneumocytes in acute lung injury. Am J Pathol 1996; 150:845-52.

2. Denekamp J: Cell kinetics and radiation biology. Int J Radiat Biol 1986;49:35780 .

3. Doherty GM, Jensen JC, Alexander HR et al. Pentoxifylline suppression of tumor necrosis factor gene transcription. Surgery 1991;110:192-8.

4. Eissner G, Kohlhuber F, Grell M et al. Critical involvement of transmembrane tumor necrosis factor ( in endothelial programmed cell death mediated by ionizing radiation and bacterial endotoxin. Blood 1995;86:4184-93.

5. Fajardo LF. Pathology of radiation injury. USA: Masson Publishing, 1982:310.

6. Fridovich I: Superoxide dismutase in biology and medicine. Pathology of Oxygen. New York: Academic Press, 1982:1-19.

7. Friesen C, Herr I, Krammer PH et al. Involvement of the CD95 (APO-1/Fas) receptor/ligand system in drug-induced apoptosis in leukemia cells. Nat Med 1996;2:574-7.

8. Fuks Z, Alfieri A, Haimovitz-Friedman A et al. Intravenous basic fibroblast growth factor protects the lung but not mediastinal organs against radiation-induced apoptosis in vivo. Cancer J Sci Am 1995;1:62.

9. Funk JO, Ernst M, Schönharting MM et al. Pentoxifylline exerts synergistic immunomodulatory effects in combination with Dexamethasone or Cyclosporin A. Int J Immunopharmacol 1995;17:1007-16.

10. Gilfillan AM, Hollingsworth M, Jones AW. The pharmacological modulation of $\left[{ }^{3} \mathrm{H}\right]$ disaturated phosphatidylcholine overflow from perifused lung slices of adult rat: a new method for the study of lung surfactant secretion. Br J Pharmacol 1983;79:363-71.

11. Gobran LI, Rooney S. Adenylate cyclase-coupled ATP receptor and surfactant secretion in type II pneumocytes from newborn rats. Am J Physiol 1997;272: L187-L196.

12. Godar DE. Light and death: photons and apoptosis. J Investig Dermatol Symp Proc 1999;4:17-23.

13. Gross NJ. The pathogenesis of radiation-induced lung damage. Lung 1981;159:115-25.

14. Guinee D, Brambilla E, Fleming $M$ et al. The potential role of bax and bcl-2 expression in diffuse alveolar damage. Am J Pathol 1997;151:999-1007.

15. Guinee DG Jr, Fleming M, Hayashi T et al. Association of p53 and WAF1 expression with in apoptosis in diffuse alveolar damage. Am J Pathol 1996;150:5318.

16. Hasleton PS. Spencer's pathology of the lung. $5^{\text {th }}$ ed., New York: McGraw-Hill, 1996:530.

17. Kastan MB, Onyekwere O, Sidransky D et al. Participation of $\mathrm{p} 53$ protein in the cellular response to DNA damage. Cancer Res 1991;51:6304-11.

18. Koo DJ, Yoo P, Cioffi WG et al. Mechanism of the beneficial effects of pentoxifylline during sepsis: maintenance of adrenomedullin responsiveness and downregulation of proinflammatory cytokines. J Surg Res 2000;91:70-6.

19. Korsmeyer SJ. Bcl-2 initiates a new category of oncogenes: regulators of cell death. Blood 1992;80:879-86.

20.Korsmeyer SJ, Yin XM, Oltvai ZN et al. Reactive oxygen species and the regulation of cell death by the Bcl-2 gene family. Biochim Biophys Acta 1995;12:63-6.

21. Noel C, Copin MC, Hazzan M et al. Immunomodulatory effect of pentoxifylline during human allograft rejection: involvement of tumor necrosis factor-alpha and adhesion molecules. Transplantation 2000;69:1102-7.

22. Mándi Y, Farkas G, Ocsovsky I et al. Inhibition of tumor necrosis factor production and ICAM - 1 expression by pentoxifylline: beneficials effects in sepsis syndrome. Res Exp Med 1995;195:297-307.

23. Murphy PG, Myers DS, Webster NR et al. Direct detection of free radical generation in an in vivo model of acute lung injury. Free Radic Res Commun 1991; 15:167-76.

24. Österreicher J, Vávrová J, Knižek J et al. Radiation pneumonitis: The model of interstitial edema. Exp Toxicol Pathol 1999;51:491-3.

25. Österreicher J, Vávrová J, Knižek J et al. Radiation pneumonitis: the influence of pentoxifylline and dexamethasone on the alveolar septal thickness and the amount of neutrophil granulocytes. Voj Zdrav Listy-Supll 2000;68:36-40.

26. Rubin P, Johnston CJ, Williams JP et al. A perpetual cascade of cytokines postirradiation leads to pulmonary fibrosis. Int $\mathbf{J}$ Radiat Oncol Biol Phys 1995;33:99-109.

27. Silva JC, Rocha MF, Lima AA et al. Effects of pentoxifylline and nabumetone on the serum levels of IL-1beta and TNFalpha in rats with adjuvant arthritis. Inflamm Res 2000;49:14-9.

28. Tanaka R: Effects of pentoxifylline on active oxygen-induced sister-chromatid exchange. J Toxicol Sci 1995;20:401-6.

29. Travis EL. The sequence of histological changes in mouse lungs after single doses of X-rays. Int J Radiat Oncol Biol Phys 1980;16:345-7.

30. Ward HE, Kemsley L, Davies L et al. The pulmonary response to sublethal thoracic irradiation in the rat. Radiat Res 1993;130:15-21. 
31. Ward WF, Kim YT, Molteni A et al. Pentoxifylline does not spare acute radiation reactions in rat lung and skin. Radiat Res 1992;129:107-11.

32. Wood KA, Youle RJ. Apoptosis and free radicals. Ann N Y Acad Sci 1994; 73:400-7.

Submitted September 2001.

Accepted November 2001.

Doc. MUDr. Jan Österreicher, Ph.D., Purkyně Military Medical Academy,

Třebešská 1575, 50002 Hradec Králové, Czech Republic. e-mail: osterreicher@pmfhk.cz. 